\title{
Numerical Study of the Heat Transfer Characteristics of Convection in Vertical Channel through FEM Simulation
}

*P. V. Janardhana Reddy

Department of Mathematics, MGIT, Hyderabad, Telangana, India

\begin{abstract}
Article Info

Volume 7, Issue 5

Page Number: 342-349

Publication Issue :

September-October-2020

\section{Article History}

Accepted : 01 Sep 2020

Published : 20 Sep 2020

An attempted is made on the free convection flow of an incompressible viscous fluid in the profile in terms of heat characteristics were analyzed. The flow is assumed to be along the axis of the channel. The uniform temperature is considered in the study. The momentum conservation equations for the fully developed flow are based on the Brinkman model. The viscous and Darcy dissipation are taken into account in the energy equation as the modification of the heat flow. By using the finite element method, the fluid and the porous matrix are in local thermal equilibrium and the flow is unidirectional along the direction of the buoyancy. The non-linear equations governing the flow, heat and mass transfer have been analyzed. Various parameters such as the velocity, temperature, concentration, Nusselt number, and Sherwood number are analyzed and their behavior is interpreted in the paper.

Keywords : Heat Transfer, Finite Element, Viscous Flow, Thermal Flow
\end{abstract}

\section{INTRODUCTION}

The heat source module is made from a highly polished stainless steel foil which is in close constant with three exposed surfaces of a stack of Bakelite strips. The study concluded that the flow and heat transfer characteristics have a strong dependence on the rate of energy input, heat source thickness and the interaction between the wakes generated by the three exposed surfaces of the heat source module. Separate correlations for average Nusselt number as a function of Grashof number, for horizontal and vertical plate, are also presented. Kelkar and Choudhury [3] carried out numerical computation of the periodically fully developed natural convection in a vertical channel with surface mounted heat generating blocks. They studied the effect of dimensionless length of the channel and module Rayleigh number on draft cooling of low power heat generating modules. Fujii et al. [4] studied, numerically and experimentally, natural convection cooling of an array of vertical finite thick parallel plates with discrete and protruding heat sources mounted on them. They proposed an optimum spacing between the plates and presented a correlation for local Nusselt number in terms of modified Grashof number. Numerical modeling of natural convection cooling of heat generating devices mounted on a vertical wall of an upright open top slot has been reported by Desrayaud and Fichera [5]. Their study highlighted the influence of design parameters such as heat generation rate, size and 
position of heat generating devices and thermal conductivity of the substrate on the heat transfer characteristics. A numerical study of steady twodimensional laminar natural convection from a single protruding heat source, mounted at mid-height of a substrate of finite thickness, was carried out by Desrayaud et al. [6].

\section{FORMULATION OF THE PROBLEM}

The flow takes place along the axis of the channel. The surface of the walls is maintained at uniform temperature. The momentum conservation equations for the fully developed flow are based on the

$$
\begin{gathered}
\frac{\partial \mathrm{u}}{\partial \mathrm{x}}=0 \\
\mu\left(\frac{\partial^{2} u}{\partial y^{2}}+\frac{\partial^{2} u}{\partial z^{2}}-\frac{u}{k}\right)+g \beta\left(T-T_{0}\right)+g \beta^{*}\left(C-C_{o}\right)=-v_{0} \frac{\partial u}{\partial y} \\
\frac{\partial^{2} T}{\partial y^{2}}+\frac{\partial^{2} T}{\partial z^{2}}+\frac{\rho_{0} v}{k_{1}}\left[\left(\frac{\partial u}{\partial y}\right)^{2}+\left(\frac{\partial u}{\partial z}\right)^{2}\right]+\frac{\rho_{0} v}{k k_{1}} u^{2}-\frac{\partial}{\partial y}\left(r q_{\partial r}\right)=-\rho_{0} c p \frac{v_{0}}{k_{1}} \frac{\partial T}{\partial y} \\
D_{1}\left(\frac{\partial^{2} C}{\partial y^{2}}+\frac{\partial^{2} C}{\partial z^{2}}\right)+K 11\left(\frac{\partial^{2} \theta}{\partial y^{2}}+\frac{\partial^{2} \theta}{\partial z^{2}}\right)=0 \\
\rho=\rho_{0}\left(1-\beta\left(\mathrm{T}-\mathrm{T}_{0}\right)-\beta^{*}\left(\mathrm{C}-\mathrm{C}_{0}\right)\right)
\end{gathered}
$$

where $\rho_{0}$ is the density at the ambient temperature $T_{\circ}$ and concentration $C_{o}$ and $\beta, k, v$ are the coefficients of Kinematic viscosity, thermal conductivity and thermal expansion of the fluid respectively, $\beta^{*}$ is the volumetric coefficient of expansion with mass fraction concentration, $\mathrm{k}$ is the permeability of the porous medium and $C_{p}$ is the specific heat at constant pressure, this the molecular diffusivity and k11is the cross diffusivity.

In view of the continuity equations, we take $\mathrm{u}=\mathrm{u}(\mathrm{y}, \mathrm{z})$

The boundary conditions are

$$
\begin{gathered}
\mathrm{u}=0 \text { on } \mathrm{z}= \pm \mathrm{b} \\
\mathrm{T}= \pm \mathrm{T}_{1}, \quad \mathrm{C}= \pm \mathrm{C}_{1}
\end{gathered}
$$

$\frac{\partial u}{\partial z}=0, \frac{\partial T}{\partial z}=0$ and $\frac{\partial C}{\partial z}=0$ on $\mathrm{Z}=0$ in view of the symmetry.

We introduce the following non-dimensional variables as follows.

$$
z^{*}=\frac{z}{b} \quad ; \quad y^{*}=\frac{y}{b} \quad ; \quad \theta^{*}=\frac{T-T_{0}}{T_{1}-T_{0}}, \quad C^{*}=\frac{C-C_{o}}{C_{1}-C_{o}},
$$




$$
u^{*}=\frac{v u}{\beta g b^{2}\left(T_{1}-T_{0}\right)}
$$

Substituting these in the governing equations the corresponding dimensionless equations under Boussinesq approximations (on dropping the asteriks) are

$$
\begin{gathered}
\frac{\partial^{2} u}{\partial y^{2}}+\frac{\partial^{2} u}{\partial z^{2}}-D^{-1} u+(\theta+N C)=-S \frac{\partial u}{\partial y} \\
\left.\frac{\partial^{2} \theta}{\partial y^{2}}+N_{2} \frac{\partial^{2} \theta}{\partial z^{2}}+G P_{1} E c\left[\left(\frac{\partial u}{\partial y}\right)^{2}+\left(\frac{\partial u}{\partial z}\right)^{2}\right)+D^{-1} u^{2}\right]=-P_{1} S \frac{\partial \theta}{\partial y} \\
\left(\frac{\partial^{2} C}{\partial y^{2}}+\frac{\partial^{2} C}{\partial z^{2}}\right)+\frac{S_{0} S c}{N}\left(\frac{\partial^{2} \theta}{\partial y^{2}}+\frac{\partial^{2} \theta}{\partial z^{2}}\right)=0
\end{gathered}
$$

For computational purpose, we choose a serendipity element with $(0,0),(0,1)(1,0)$ and $(1,1)$ as its vertices. The eight nodes of the element are shown in Fig.(a) and the quadratic interpolation functions at these nodes are

$$
\begin{array}{llll}
N_{1}=-2 \quad(y-1)(z-1)\left(z+y-\frac{1}{2}\right) & ; & N_{2}=-4 z(z-1)(y-1) \\
N_{3}=-2 \quad z \quad(y-1) \quad\left(z-y+\frac{1}{2}\right) & ; & N_{4}=-4 \quad y \quad z \quad(y-1) \\
N_{5}=2 \quad y \quad z \quad\left(z+y-\frac{3}{2}\right) & ; & N_{6}=-4 \quad y \quad z(z-1) \\
N_{7}=2 \quad y \quad(z-1)\left(z-y+\frac{1}{2}\right) & ; & N_{6}=-4 \quad y \quad(z-1)(y-1)
\end{array}
$$

Substituting these shape functions in (3.19) and integrating over the element domain the matrix for the global nodes of $u$ viz. $u_{i}(i=1,2, \ldots \ldots)$ reduces to a $8 \times 8$ matrix equations.

This $8 \times 8$ matrix equations can be partitioned in the form

$$
\left[\begin{array}{ll}
A^{11} & A^{12} \\
A^{21} & A^{22}
\end{array}\right]\left[\begin{array}{l}
\Delta_{U}^{1} \\
\Delta_{U}^{2}
\end{array}\right]=\left[\begin{array}{c}
F_{U}^{1} \\
F_{U}^{2}
\end{array}\right]
$$

where $\Delta_{\mathrm{U}}^{1}, \Delta_{\mathrm{U}}^{2}, \mathrm{~F}_{\mathrm{U}}^{1}, \mathrm{~F}_{\mathrm{U}}^{2}$ are column matrices given by

$$
\Delta_{\mathrm{U}}^{1}=\left[\begin{array}{c}
\mathrm{U}_{1} \\
\mathrm{U}_{2} \\
\mathrm{U}_{3} \\
\mathrm{U}_{4}
\end{array}\right] \quad, \quad \Delta_{\mathrm{U}}^{2}=\left[\begin{array}{c}
\mathrm{U}_{5} \\
\mathrm{U}_{6} \\
\mathrm{U}_{7} \\
\mathrm{U}_{8}
\end{array}\right]
$$

equation (3.20) yields the following two equations in terms of the partitioned matrices. 


$$
\begin{aligned}
& {\left[S^{11}\right]\left[\Delta_{U}^{1}\right]+\left[S^{12}\right] \quad\left[\Delta_{U}^{2}\right]=\left[F_{U}^{1}\right]} \\
& {\left[S^{21}\right] \quad\left[\Delta_{U}^{1}\right]+\left[S^{22}\right]} \\
& {\left[\Delta_{U}^{2}\right]=\left[F_{U}^{2}\right]}
\end{aligned}
$$

Similarly the $8 \times 8$ matrix equations for $\theta_{j}, C_{j}(j=1,2, \ldots ., 8)$ in the partitioned form are

$$
\begin{aligned}
& {\left[\begin{array}{ll}
B^{11} & B^{12} \\
B^{21} & B^{22}
\end{array}\right]\left[\begin{array}{l}
\Delta_{\theta}^{1} \\
\Delta_{\theta}^{2}
\end{array}\right]=\left[\begin{array}{c}
F_{\theta}^{1} \\
F_{\theta}^{2}
\end{array}\right]} \\
& {\left[\begin{array}{ll}
L^{11} & L^{12} \\
L^{21} & L^{22}
\end{array}\right] \quad\left[\begin{array}{l}
\Delta_{C}^{1} \\
\Delta_{C}^{2}
\end{array}\right]=\left[\begin{array}{c}
F_{C}^{1} \\
F_{C}^{2}
\end{array}\right]}
\end{aligned}
$$

where $\Delta_{\theta}^{1}, \Delta_{\theta}^{2}, \mathrm{~F}_{\theta}^{1}, \mathrm{~F}_{\theta}^{2}, \Delta_{\mathrm{C}}^{1}, \Delta_{\mathrm{C}}^{2}, \mathrm{~F}_{\mathrm{C}}^{1}, \mathrm{~F}_{\mathrm{C}}^{2}$ are column matrices given by

$$
\Delta_{\theta}^{1}=\left[\begin{array}{l}
\theta_{1} \\
\theta_{2} \\
\theta_{3} \\
\theta_{4}
\end{array}\right] \quad ; \quad \Delta_{\theta}^{2}=\left[\begin{array}{l}
\theta_{5} \\
\theta_{6} \\
\theta_{7} \\
\theta_{8}
\end{array}\right] \quad ; \quad \Delta_{C}^{1}=\left[\begin{array}{l}
C_{1} \\
C_{2} \\
C_{3} \\
C_{4}
\end{array}\right] \quad ; \quad \Delta_{C}^{2}=\left[\begin{array}{l}
C_{5} \\
C_{6} \\
C_{7} \\
C_{8}
\end{array}\right]
$$

The boundary conditions (essential) on the primary variables are

$$
\begin{aligned}
& \mathrm{u}_{3}=\mathrm{u}_{4}=\mathrm{u}_{5}=0 ; \\
& \theta_{3}=\theta_{4}=\theta_{5}=1 \text { and } \\
& \mathrm{C}_{3}=\mathrm{C}_{4}=\mathrm{C}_{5}=1 \quad \text { on } \quad \mathrm{y}=1
\end{aligned}
$$

In view of the symmetry conditions we obtain

$$
\begin{aligned}
& \mathrm{Q}_{1}=\mathrm{Q}_{2}=\mathrm{Q}_{6}=\mathrm{Q}_{7}=\mathrm{Q}_{8}=0 \\
& Q_{1}^{T}=Q_{2}^{T}=Q_{6}^{T}=Q_{7}^{T}=Q_{8}^{T}=0 \\
& Q_{1}^{C}=Q_{2}^{C}=Q_{3}^{C}=Q_{4}^{C}=Q_{8}^{C}=0
\end{aligned}
$$

Solving the ultimate $8 \times 8$ matrices we determine the unknown global nodal values of $u_{i}, \theta_{i}(i=1,2, \ldots \ldots ., 8)$.

The solution for $\mathrm{u}, \theta$ may now be represented as

$$
u=\sum_{k=1}^{8} u_{i} N_{i}, \theta=\sum_{j=1}^{8} \theta_{j} N_{j} \text { and } C=\sum_{i=1}^{8} C_{i} N_{i}
$$

The shear stress on the boundary $\mathrm{y}=1$ in the non-dimensional form is given by

$$
\tau=\left(\frac{\partial u}{\partial y}\right)_{y=1}
$$

The rate of heat and mass transfer in the non-dimensional form on the boundary is

$$
N u=\left(\frac{\partial \theta}{\partial y}\right)_{y=1} \quad, \quad S h=\left(\frac{\partial C}{\partial y}\right)_{y=1}
$$

The shear stress, the Nusselt number and Sherwood number are evaluated computationally for variations in the governing parameters. 


\section{DISCUSSION OF NUMERICAL RESULTS}

The values of $\mathrm{u}$ at $\mathrm{y}=0$ are higher than those at $\mathrm{y}=1$ level for all variations in parameters. Figures $1,2,3,4$ and 5 represents the variation of non-dimensional temperature at the horizontal levels $y=0$ \& $1 / 2$ with respect to $\mathrm{G}, D^{-1}, \alpha$, Ec and N. It is found that the non-dimensional temperature is positive for all variations. Figures $3 \& 4$ represent $\theta$ with Grashof number $G$. An increase in $G$ results in a depreciation in $\theta$ at $\mathrm{y}=0 \& 1 / 2$. It is found that the actual temperature enhances with increase in $D^{-1}$.

Thus lesser the permeability of the porous medium larger the actual temperature at all horizontal levels. The influence of heat source on $\theta$ is exhibited in figures. We find a marginal depreciation in $\theta$ with increase in the heat source parameter $\alpha$. The variation of $\theta$ with radiation parameter N1 shows that an increase in $\mathrm{N} 1$ leads to an enhancement in the actual temperature at $\mathrm{y}=0 \& 1$ levels. The variation of $\theta$ with $G, D^{-1}, \alpha, E c$ and $N$, at the vertical levels $Z=0 \& 1 / 2$ is shown in figures 3,4 and 5 . The variation of $\theta$ with Grashoff number $G$ is shown in figures $15 \& 16$ at the vertical levels Z=0\&1/2. From this figures we notice that the actual temperature depreciates with increase in $G$ and enhances with $D^{-1}$.

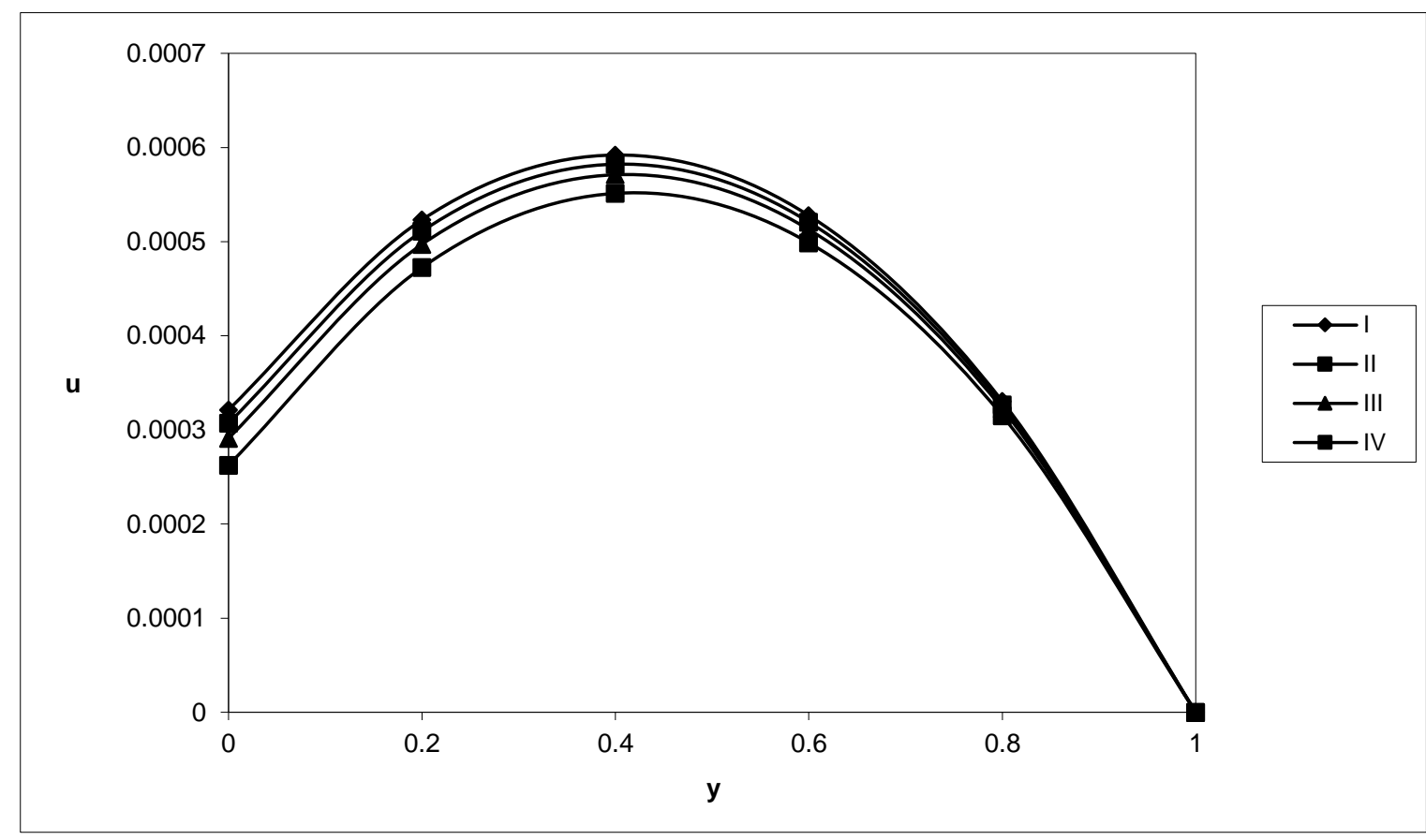

Fig. 1:

Variation of $\mathrm{u}$ with $\mathrm{G}$ at $\mathrm{y}=0.5$ level

$\mathrm{M}=5 ; \mathrm{G}=200 ; \mathrm{cn}=0.5 ; \mathrm{S}=0.8 ; \mathrm{k}=0.5 ; \mathrm{qe}=0.71 ; \mathrm{d}=2000$;

$\begin{array}{ccccc}\text { G } & \text { II } & \text { III } & \text { IV } \\ & 10 & 50 & 100 & 200\end{array}$


P. V. Janardhana Reddy Int J Sci Res Sci \& Technol. September-October-2020; 7 (5) : 342-349

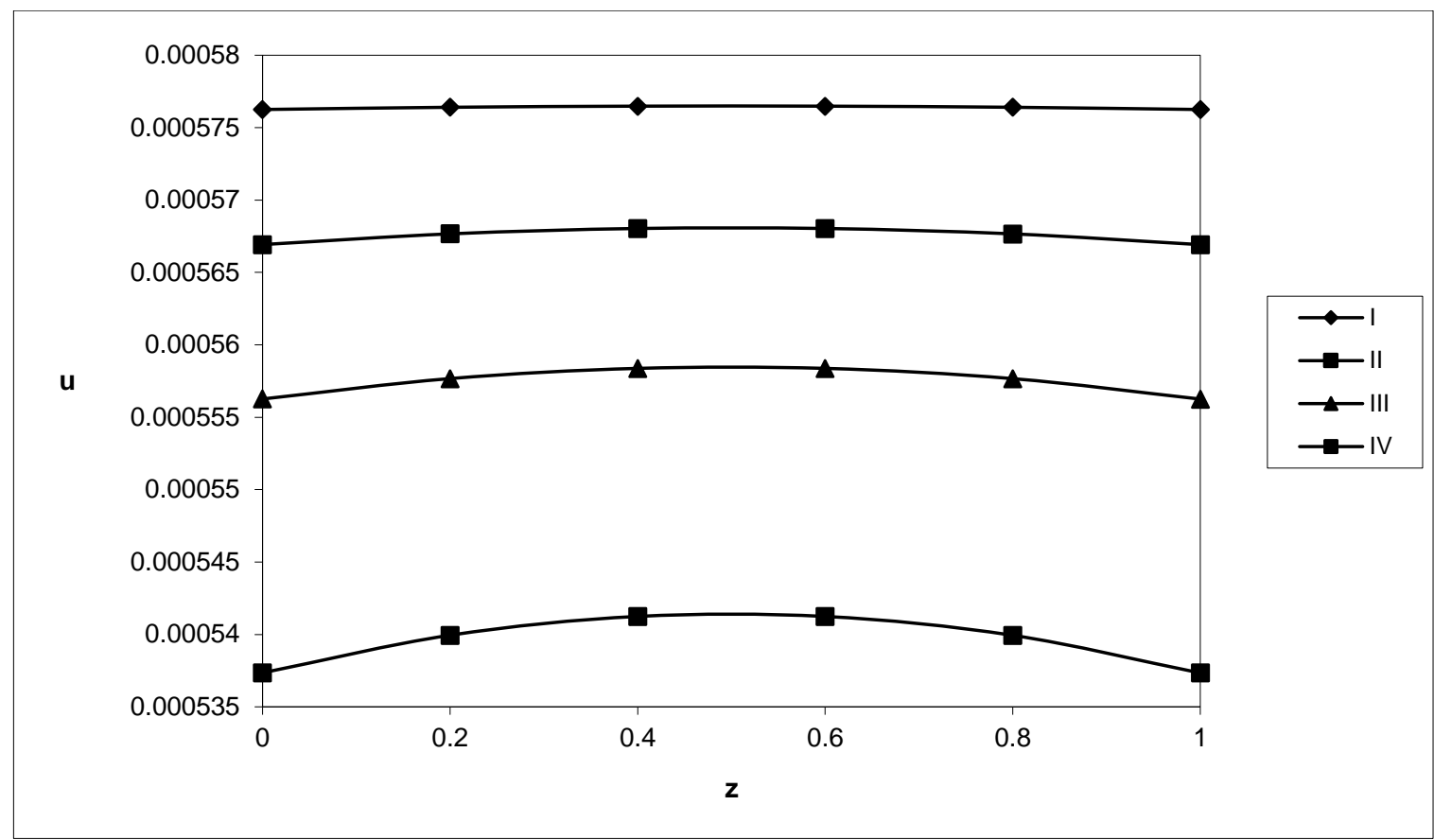

Fig. 2:

Variation of $\mathrm{u}$ with $\mathrm{G}$ at $\mathrm{z}=0.5$ level

$\mathrm{M}=5 ; \mathrm{G}=200 ; \mathrm{cn}=0.5 ; \mathrm{S}=0.8 ; \mathrm{k}=0.5 ; \mathrm{qe}=0.71 ; \mathrm{d}=2000$;

I II III IV

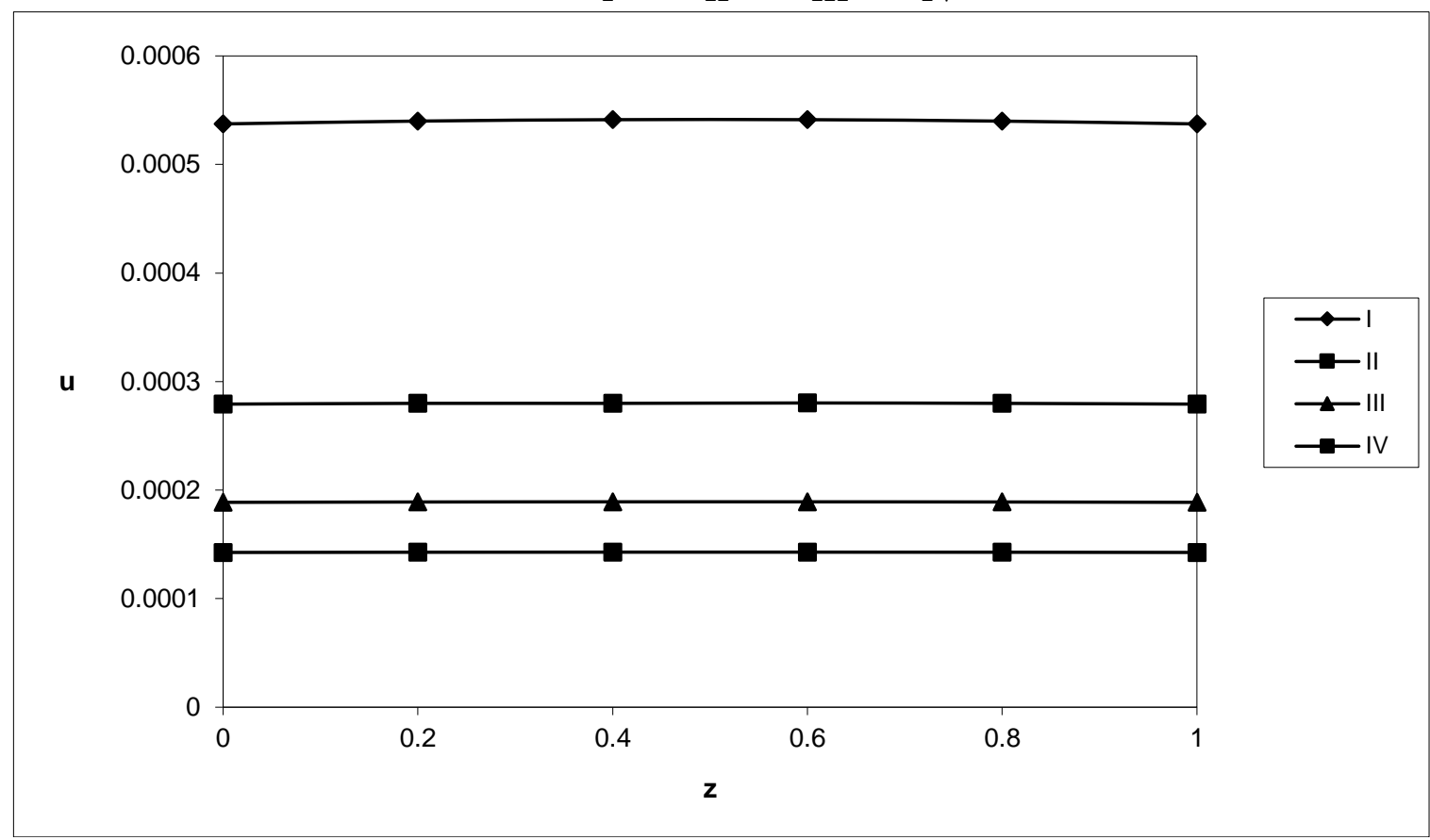

Fig. 3:

Variation of $\mathrm{u}$ with $\mathrm{D}^{-1}$ at $\mathrm{z}=0.5$ level

$\mathrm{M}=5 ; \mathrm{G}=200 ; \mathrm{cn}=0.5 ; \mathrm{S}=0.8 ; \mathrm{k}=0.5 ; \mathrm{qe}=0.71 ; \mathrm{d}=2000 ; \mathrm{z}=0.5$

I II III IV

$D^{-1} \quad 2 \times 10^{3} \quad 4 \times 10^{3} \quad 6 \times 10^{3} \quad 8 \times 10^{3}$ 


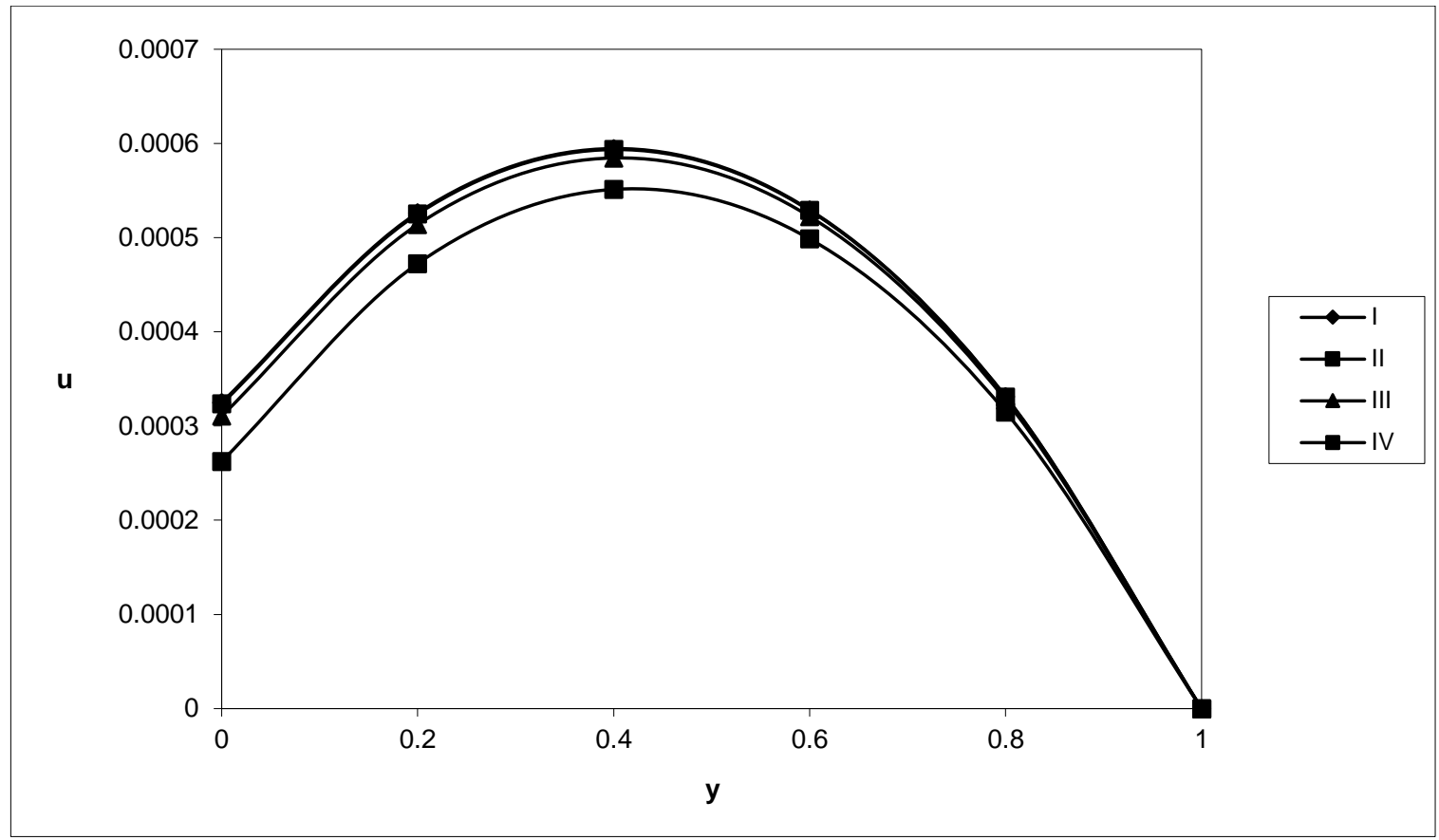

Fig. 4:

Variation of $\mathrm{u}$ with Ec at $\mathrm{y}=\mathbf{0 . 5}$ level

$\mathrm{M}=5 ; \mathrm{G}=200 ; \mathrm{cn}=0.5 ; \mathrm{S}=0.8 ; \mathrm{k}=0.5 ; \mathrm{qe}=0.71 ; \mathrm{d}=2000$;
I II III IV
$\begin{array}{lllll}\text { Ec } & 0 & 0.01 & 0.1 & 0.5\end{array}$

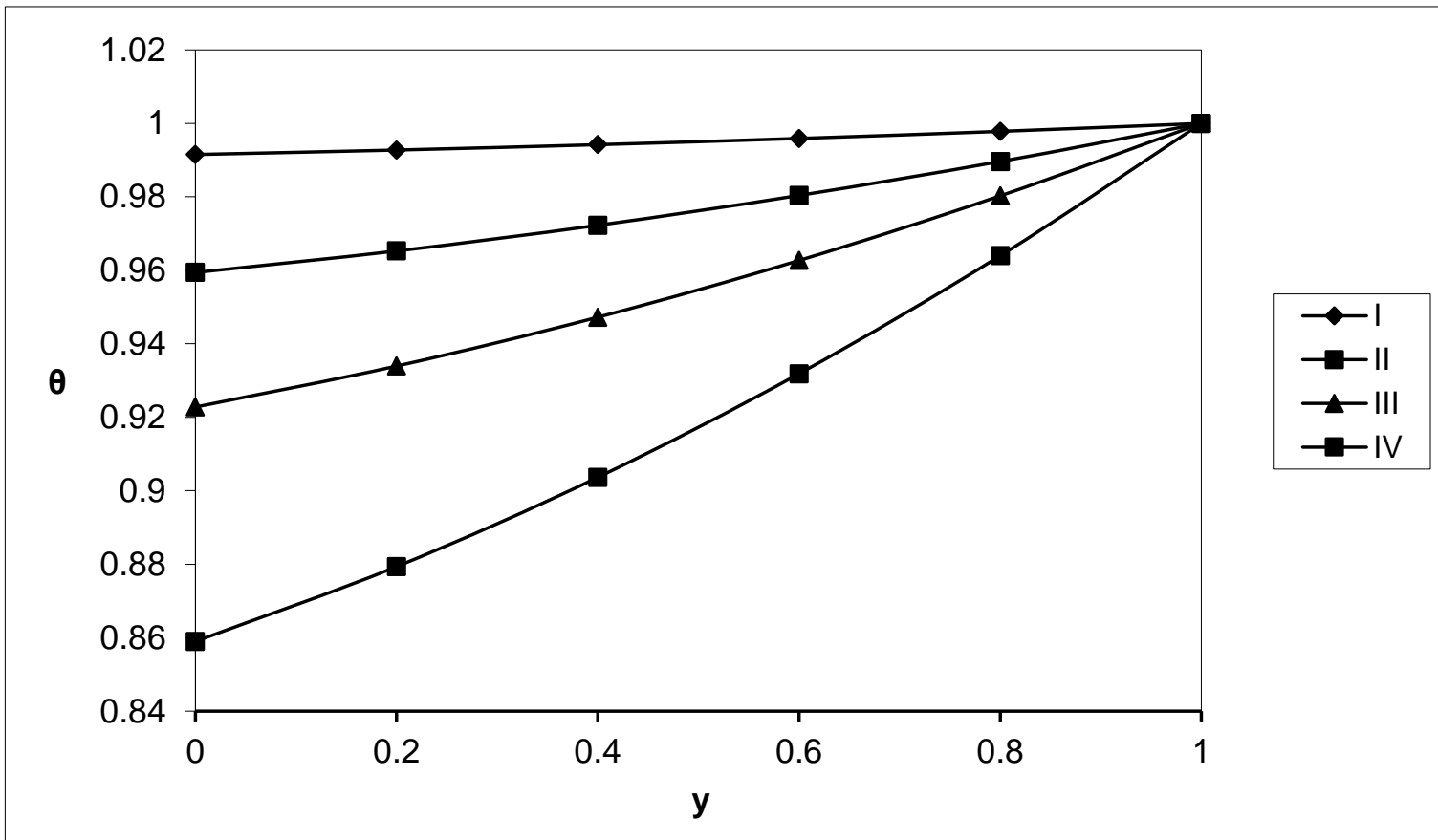

Fig. 5:

Variation of $\theta$ with $\mathrm{G}$ at $\mathrm{y}=0$ level

$\mathrm{M}=5 ; \mathrm{G}=200 ; \mathrm{cn}=0.5 ; \mathrm{S}=0.8 ; \mathrm{k}=0.5 ; \mathrm{qe}=0.71 ; \beta=2 ; \mathrm{d}=2000$
G $\quad 10 \quad 50 \quad 100 \quad 200$
II
III IV 


\section{REFERENCES}

[1]. Fujii, M, Gima S,Tomimura T, Zhang, X: Natural convection to air from an array of vertical parallel plates with discrete and protruding heat sources. Int J Heat Fluid Flow 17, pp 483-490, (1996).

[2]. Desrayaud, G, \& Fichera, A : On Natural convective heat transfer in vertical channels with a single surface mounted heat flux module. ASME J Heat Transfer 125, pp 734739 (2003).

[3]. Ermolaev, A and Zhbanov, A.I.: Mixed convection in vertical channel with Discrete Heat Sources at the wall. Fluid Dynamics, Vol.44, No.4, pp. 511-516. (2009).

[4]. Barletta and Nield,D.A.: Combined forced and free convective flow in a vertical porous channel: The effects of viscous dissipation and pressure work. Transp Porous Med 79:319334, (2009).

[5]. Doshy, I: Reliability impact of thermal design. In: Technical conference-IEPS, 4th annual international electronic packaging conference, pp 307-317,(1984).

[6]. Kang, BH, Jaluria, Y: Natural convection heat transfer characteristics of a protruding thermal source located on horizontal and vertical surface. Int J Heat Mass Transfer 33, pp 1347-1357, (1990).

[7]. Kelkar, M K, \& Choudhury, D: Numerical prediction of periodically fully developed natural convection in vertical channel with surface mounted heat generating blocks. Int J Heat Mass Transfer 36, pp 1133-1145, (1993).

[8]. Doshy, I: Reliability impact of thermal design. In: Technical conference-IEPS, 4th annual international electronic packaging conference, pp 307-317,(1984).

\section{Cite this Article}

P. V. Janardhana Reddy, "Numerical Study of the Heat Transfer Characteristics of Convection in Vertical Channel through FEM Simulation", International Journal of Scientific Research in Science and Technology (IJSRST), Online ISSN : 2395-602X, Print ISSN : 2395-6011, Volume 7 Issue 5, pp. 342-349, September-October 2020. Available at doi : https://doi.org/10.32628/IJSRST196211 Journal URL : http://ijsrst.com/IJSRST196211 